\title{
Espectro clínico de la infección en niños por virus SARS-CoV-2 en un centro de referencia pediátrico en plena pandemia. Reporte del Comité Clínico COVID, Hospital de Niños Roberto del Río, Santiago Chile
}

\author{
Clinical manifestations of SARS-CoV-2 infection in children in the middle of pandemic \\ season in a pediatric tertiary center. Report of local COVID Clinical Committee, \\ Hospital de Niños Roberto del Río, Santiago Chile
}

Fernanda Cofré1, Jorge Mackenney ${ }^{1,2,3}$, Cecilia Poli1,4,5, Maryel Riquelme1, Cristian Carvajal', Patricia Álvarez 1,2,4, Valeria Acevedo ${ }^{1,2,4}$, M. Lidia Valenzuela', Patricia Verdugo 1,2,6, Mónica Varas ${ }^{2,4,7}$ y Lorena Tapia 2,3

${ }^{1}$ Hospital de Niños Roberto del Río. Santiago, Chile.

${ }^{2}$ Facultad de Medicina, Universidad de Chile. Santiago, Chile

${ }^{3}$ Clínica Las Condes. Santiago, Chile.

${ }^{4}$ Clínica Alemana de Santiago. Santiago, Chile

${ }^{5}$ Instituto de Ciencia e Innovación en Medicina, Universidad del Desarrollo. Santiago, Chile.

${ }^{6}$ Clínica Santa María. Santiago, Chile.

${ }^{7}$ Hospital San Juan de Dios. Santiago, Chile.

Financiamiento: no tiene.

Los autores declaran ausencia de conflictos de interés.

Recibido: 23 de julio de 2020 / Aceptado: 20 de diciembre de 2020

\section{Resumen}

Introducción: La infección por virus SARS-CoV-2 responsable de la pandemia actual, es una entidad clínica y fisiopatológica nueva y en desarrollo, cuyo control aún es incierto mientras no contemos con una vacuna efectiva y de distribución universal. Descrita inicialmente como una enfermedad respiratoria mayoritariamente de adultos, los niños también pueden enfermar y se ha visto que en ellos las manifestaciones clínicas de enfermedad suelen diferir a las de los adultos expresándose como cuadros benignos en su mayoría. Si requieren hospitalización o algún tipo de asistencia, el cuadro se resuelve con tratamiento de soporte y sin complicaciones, mayoritariamente. Sin embargo, en el síndrome inflamatorio multisistémico asociado a COVID-19 (SIM-C) es de vital importancia la sospecha precoz y la derivación a un centro de alta complejidad para otorgar el soporte y tratamiento adecuado para lograr una buena y adecuada sobrevida. Objetivo: Describir el espectro clínico de enfermedad por virus SARS$\mathrm{CoV}-2$ en un centro de referencia pediátrico con la pandemia aún en desarrollo. Método: Se presenta la casuística de 537 pacientes con infección por SARS-CoV-2 atendidos entre marzo 1 y julio 15, 2020, con descripción de aquellos que fueran hospitalizados. Resultados: $127(23 \%)$ de ellos fueron internados y de éstos $69 \%$ sintomáticos. Veintiséis pacientes (20\%) de los hospitalizados presentaron SIM-C y sólo uno falleció por complicaciones de sus patologías de base.

Palabras clave: COVID-19; síndrome inflamatorio multisistémico asociado a COVID-19; pandemia.

\section{Abstract}

Background: SARS-CoV-2 virus infection responsible for de pandemic in course, is a new clinical and physiopathological entity, whose control is still uncertain till we can provide an effective and universal vaccine. In the beginning it was described as a respiratory disease which affects mainly adults, children can have the disease too and in this group the disease can be different than the adult disease. Acute infection in children is mostly mild and when it requires hospital assistance it resolves with support therapy and without complications most of the time. However, in the Pediatric Inflammatory Multisystemic Syndrome is vital the early clinical suspect and refers to a tertiary center to bring support and properly treatment. Aim: To describe the clinical spectrum of SARS-CoV-2 virus disease in a pediatric referral center with the pandemic still in development. Method: A case series of 537 patients with SARS-CoV-2 infection treated between March 1 and July 15, 2020 is presented with a description of those who were hospitalized. Results: 127 (23\%) of them were hospitalized and of these $69 \%$ were symptomatic. Twenty-six patients $(20 \%)$ of those hospitalized presented PIMS, only one died for complications of his chronic diseases.

Key words: COVID-19; Pediatric Inflammatory Multisystemic Syndrome; pandemic. 
espectro clínico de enfermedad por virus SARS-CoV-2 en un centro de referencia pediátrico en Santiago de Chile, con la pandemia aún en desarrollo.

En diciembre 2019 se describe en Wuhan, China una epidemia de neumonía grave por un agente desconocido lográndose en poco tiempo identificar como responsable al coronavirus SARS-CoV-2 cuya alta contagiosidad y rol pandémico ha sido ampliamente documentado a la fecha y se encuentra en pleno desarrollo ${ }^{1,2}$. El primer caso de infección por SARS-CoV-2 en Chile se identificó en marzo 2020, correspondiendo a un adulto proveniente de un viaje al extranjero. Pocas semanas más tarde, al perderse la trazabilidad local, se inicia la fase 4 definida por la OMS para el control de la pandemia ${ }^{3}$.

En comparación a los de casos de enfermedad por coronavirus SARS-CoV-2 (COVID-19) en adultos, la población pediátrica se describe como un grupo relevante en la transmisión comunitaria, pero de bajo riesgo de enfermar gravemente y con un espectro clínico de enfermedad predominantemente de tipo respiratorio ${ }^{4,5}$. Sin embargo, con la progresión de infectados en número y extensión y con el avance del conocimiento de nuevos casos en Europa, se evidenció que, si bien los niños no enferman como los adultos, existe un espectro clínico bastante más amplio y heterogéneo que incluye fiebre, sintomatología respiratoria y/o gastrointestinal frecuente ${ }^{6,7}$ y cuyo fenotipo más grave y característico corresponde al síndrome inflamatorio multisistémico asociado a $\mathrm{CO}$ VID-19 (SIM-C), el que ha generado alerta mundial ${ }^{8-13}$.

El Hospital de Niños Roberto del Río (HNRR) es uno de los tres hospitales exclusivamente pediátricos en Chile y funciona como centro de referencia nacional para patologías oncológicas, cardiovasculares, neuro-quirúrgicas, grandes quemados y politraumatismos en pacientes bajo 15 años. Tiene una población asignada de 1.167.285 habitantes de la cual 160.746 tienen menos de 15 años según el Instituto Nacional de Estadísticas (INE) 2019.

Desde mediados de abril del 2020 y bajo indicación de la Dirección del HNRR, se conformó un Comité Clínico Multidisciplinario e Institucional (Comité COVID-19), cuya función principal ha sido apoyar y guiar el manejo clínico de los pacientes hospitalizados por COVID-19. El comité está formado por pediatras infectólogas, inmunóloga, intensivistas, broncopulmonar, cardiólogas, hematóloga y hemato-oncóloga. A través de sesiones virtuales diarias junto a los médicos tratantes y los residentes de turno, este comité ha evaluado los casos COVID-19, revisado la literatura científica, expuesto en Reuniones Clínicas y propuesto las guías de manejo clínico institucionales. Por otro lado, a contar del 27 de abril de 2020, se implementó el tamizaje COVID-19 universal mediante RPC SARS-CoV-2 a todo paciente ingresado a hospitalización, independiente de la causa o unidad de ingreso.

El siguiente reporte tiene como objetivo describir el

\section{Pacientes y Métodos}

Para este reporte, se realizó un análisis retrospectivo de los casos de pacientes bajo 15 años, consultantes al HNRR, en el Servicio de Urgencia, en Atención Ambulatoria Institucional y hospitalizados, en los que se encontró una reacción de polimerasa en cadena (RPC) para virus SARS-CoV-2 positiva en el periodo 1 de marzo al 15 de julio de 2020.

La búsqueda de casos se realizó en base a los resultados del laboratorio de microbiología (Sistema Informático R.E.A.L. ${ }^{\circledR}$, Biomerieux, Francia), mediante el sistema de vigilancia y notificación obligatoria EPIVIGILA, MINSAL a cargo de la Unidad de Epidemiología local y mediante la revisión de casos evaluados por el Comité Clínico COVID institucional.

Se separó a los pacientes evaluados en dos grupos: pacientes con RPC SARS-CoV-2 (+) en el tamizaje de ingreso o durante la hospitalización y pacientes con RPC SARS-CoV-2 (+) o (-) y que presentaron un cuadro clínico compatible con el SIM-C, una vez que fueron evaluados por el Comité Clínico COVID institucional.

El análisis de los datos es de tipo descriptivo y se contó con la autorización del Director de la institución para su publicación.

\section{Resultados}

Se identificó un total de 537 pacientes con detección de SARS-CoV-2 por RPC. El promedio de edad fue de 44 meses con un rango 0 a 211 meses y $57 \%$ son de sexo masculino. $410(77 \%)$ de los pacientes fueron atendidos de forma ambulatoria, mientras 127 pacientes fueron internados o ya estaban hospitalizados por otra causal al momento del diagnóstico (Figura 1).

\section{Pacientes hospitalizados con RPC (+) (n: 127)}

Ochenta y ocho casos (69\%) evolucionaron con enfermedad por COVID-19 y en 39 (31\%) el hallazgo se realizó en relación con el tamizaje universal y evolucionaron en forma asintomática respecto a la infección por COVID-19. De ellos, 94 casos (74\%) requirieron hospitalización en una Unidad de Pediatría Médico Quirúrgica (UMQ) y $33(26 \%)$ requirieron hospitalización en una Unidad de Cuidados Intensivos (UCI).

Co-morbilidades. Cuarenta y seis (36,2\%) presentaban patologías de base: 11 con cardiopatías congénitas, 7 con patología oncológica, 4 con patología neurológica, 4 con 


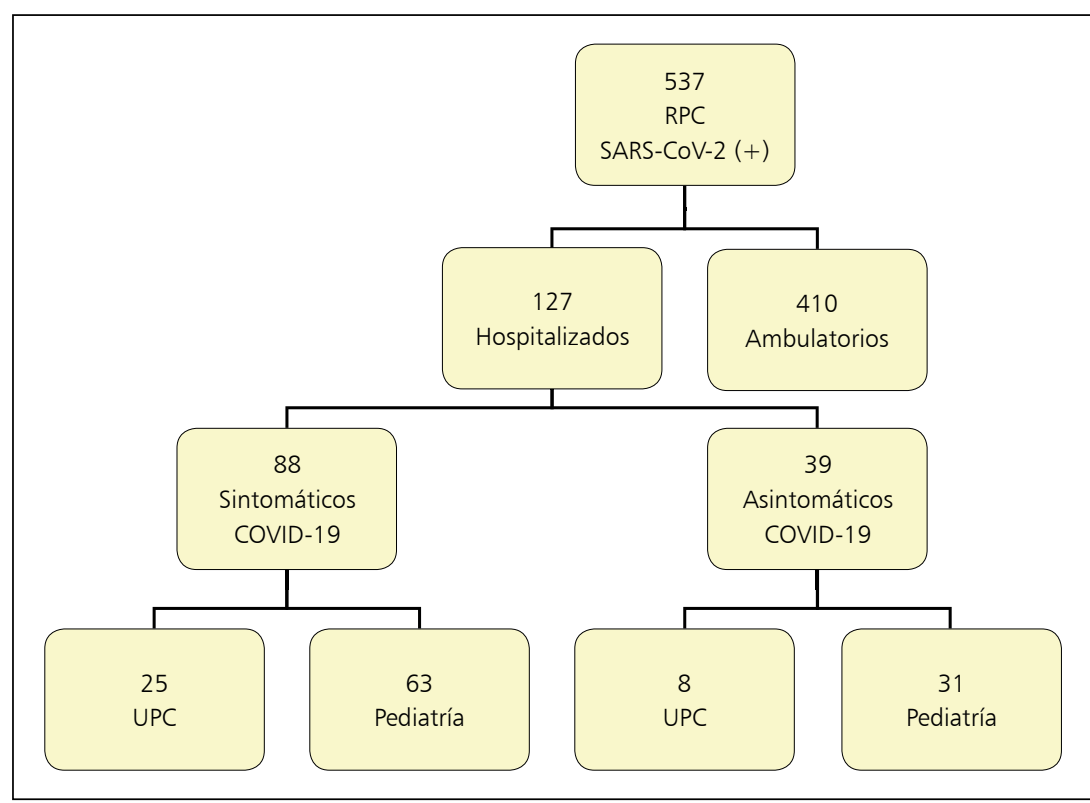

Figura 1. Pacientes pediátricos con RPC SARS-CoV-2 (+) evaluados en pandemia de COVID-19 en el Hospital de Niños Roberto del Río.

Tabla 1. Características de los 127 pacientes hospitalizados con RPC COVID (+) en el Hospital de Niños Roberto del Río

n (\%)

\section{Edad (meses)}

Sexo masculino (\%)

59

$75(59)$

Co-morbilidad

$46(36,2)$

Enfermedad por COVID-19

- Síndrome febril

- Virosis respiratoria

- Neumonía, bronconeumonía

- Síntomas gastrointestinales

Perniosis

- SIM-C

- Otros*

(cetoacidosis diabética, s. confusional, s. nefrótico, crisis suprarrenal, ALTE,

adenitis, sinovitis, neutropenia febril, s. convulsivo, vólvulo intestinal)

\section{Pacientes asintomáticos}

- Patología quirúrgica

Patología traumatológica

Patología oncológica

Patología pediátrica

Patología psiquiátrica

Caso social

Servicio de hospitalización

- Médico-quirúrgico

- Unidad de Cuidados Intensivos

$94(74)$

$33(26)$

Mortalidad

$1(0,78)$ enfermedad pulmonar crónica, 4 con inmunodeficiencia primaria o secundaria y 12 con otras patologías (diabetes mellitus, malformación genito-urinaria, cirrosis hepática, intestino corto). Pese a ello, sólo 6/46 (13,0\%) de estos pacientes requirieron traslado a UCI o evolucionaron como COVID-19 grave.

Las manifestaciones clínicas observadas en los pacientes hospitalizados con COVID-19 fueron: el síndrome febril $(17 \%)$, virosis respiratoria alta $(9 \%)$, neumonía o bronconeumonía ( $9 \%$ ) y síntomas gastrointestinales (4,5\%) (Tabla 1).

Tres pacientes recibieron hidroxicloroquina como terapia para la infección por SARS-CoV-2.

Cabe mencionar que sólo se presento el fallecimiento de un paciente, portador de múltiples patologías, que presentó una bronconeumonía grave con RPC (+) y que se le otorgó tratamiento y soporte proporcional a su condición de base.

\section{Pacientes con SIM-C (n: 26)}

Veintiséis pacientes presentaron cuadros clínicos compatibles con la definición de SIM-C $\left({ }^{12}\right)$, lo que corresponde a un $20,4 \%$ de los pacientes hospitalizados con RPC (+). En 12 (46\%) de ellos el diagnóstico de infección por SARS-CoV-2 se confirmó con RPC $(+)$; en $10(38 \%)$ casos se encontró $\operatorname{IgM}$ y/o $\operatorname{IgG}(+)$ y en los 4 (15\%) restantes sólo existió el antecedente epidemiológico de contacto con un caso confirmado.

Fenotípicamente los pacientes se presentaron clínicamente como Kawasaki like en 13 (50\%) casos, shock vasopléjico en $11(42 \%)$ pacientes y miocarditis pura en $2(7,7 \%)$ pacientes.

En los 26 pacientes de este grupo se obtuvo evaluación ecocardiográfica, encontrándose normal en 15 (58\%) de ellos; los hallazgos patológicos en los 11 pacientes $(42 \%)$ restantes se detallan en la Tabla 2. Durante la hospitalización, $20(77 \%)$ pacientes requirieron estadía en UCI. De ellos, 18 (69\%) recibieron inmunoglobulina endovenosa, en $15(58 \%)$ pacientes se utilizó tratamiento corticoesteroidal, un caso $(3,8 \%)$ recibió plasma de convaleciente y dos casos $(7,6 \%)$ recibieron tocilizumab. No hubo mortalidad en el grupo de pacientes con SIM-C en el periodo observado. (Tabla 2).

\section{Discusión}

Nuestro centro hospitalario incluye 4 de las 30 comunas con más casos acumulados a nivel país y tal como se esperaba, ha recibido y diagnosticado un número importante de casos de infección por virus SARS-CoV-2 en población pediátrica; con una incidencia global de $0,3 \%$ para la población menor de 15 años asignada.

El espectro clínico de los pacientes hospitalizados

*Otros: examen de RPC solicitado por protocolo al ser hospitalizado. 
síndrome de Kawasaki atípico. Este último, a diferencia de la enfermedad de Kawasaki clásica, se presenta en población pediátrica de mayor edad a lo habitual y sin necesidad de que estén presentes todos los elementos de la enfermedad de Kawasaki clásico ${ }^{18-20}$.

En los casos de SIM-C evaluados en nuestro centro hospitalario, el mayor compromiso y sintomatología cardiovascular y sistémico implicaron que cerca de $80 \%$ de los casos requieren monitorización y tratamiento en una unidad de cuidados intensivos.

La terapia aplicada en los casos de SIM-C refleja la escasa evidencia de cuál es la mejor opción para utilizar en este síndrome.

La discusión diaria y multidisciplinaria de estos pacientes, junto con una revisión exhaustiva y constante de la literatura científica reciente nos ha permitido distinguir localmente cuatro fenotipos clínicos: SIM-C sin shock ni síntomas de Kawasaki; SIM-C con shock; SIM-C con compromiso cardiovascular (compromiso miocárdico, pericárdico, de arterias coronarias y valvular); SIM-C Kawasaki-like. Una detección temprana de los distintos fenotipos clínicos puede guiar las intervenciones terapéuticas y el seguimiento de estos pacientes, considerando idealmente un manejo multidisciplinario en toda la evolución. En nuestra cohorte, el 100\% de los pacientes que cursaron con SIM-C pudo acceder a esta evaluación multidisciplinaria, incluyendo evaluación cardiovascular, hematológica, de intensivo pediátrico, infectológica, inmunológica, y broncopulmonar, lo que asociado a tratamientos de soporte y específicos oportunos creemos ha permitido dar cuenta de los resultados observados.

Finalmente, queremos hacer énfasis que en ambientes de alta circulación viral es de vital importancia la sospecha precoz de estas entidades, la derivación de los pacientes a un centro de alta complejidad para poder dar el soporte y tratamiento adecuado, lo que a nuestro juicio ha permitido la excelente sobrevida en nuestra población.

Una limitante al analizar esta serie ha sido que la pandemia se encuentra aún en desarrollo por lo que los resultados clínicos, epidemiológicos y/o de mortalidad podrían variar en el tiempo. Por otro lado, la información y la evidencia disponible están aún en desarrollo, haciendo variar las conductas diagnósticas y terapéuticas, lo que se reflejó en que no todos los pacientes contaron con la misma disponibilidad de recursos diagnósticos y/o terapéuticos al momento de su enfermedad. Lo que inicialmente podría haber estado recomendado y ser promisorio como fue el uso de hidroxicloroquina, ya no cuenta con evidencia a favor o, por el contrario, pacientes que fueran complejos de abordar inicialmente, en la medida que ha existido mayor y mejor conocimiento y experiencia, se logran apoyar de manera mas eficiente y eficaz. Los resultados a mediano a largo plazo aún están por verse. 


\section{Conclusiones}

En la enfermedad por COVID-19, el espectro clínico de los pacientes pediátricos hospitalizados es amplio, con manifestaciones clínicas que van desde portadores asintomáticos a sintomatología pediátrica menor, frecuente, y el SIM-C, del cual aún estamos aprendiendo.

La mayoría de los niños presenta una evolución clínica benigna, con respuesta rápida y favorable al tratamiento sintomático; sin embargo, en el SIM-C la mayoría de los casos requiere de cuidados intensivos y tratamiento inmunomodulador por el grave compromiso multisistémico que implica un riesgo vital.

En ambientes de alta circulación viral es de vital importancia la sospecha precoz y el tamizaje para poder brindar una atención adecuada y segura.

\section{Autores, y mails de contactos}

Dra. Fernanda Cofré Segovia

fmcofres@gmail.com

Dra. Lorena Tapia Faúndez

lorenaisabeltapia@gmail.com
Dra. Jorge Mackenney

jmackenney@clc.cl

Dra. Cecilia Poli Harlowe

cecipolih@gmail.com

Dra. Maryel Riquelme

Maryel.riquelme@redsalud.gov.cl

Dr. Cristian Carvajal

cmcctaz@gmail.com

Dra. Patricia Alvarez Zenteno

palvaerzz@alemana.cl

Dra. Valeria Acevedo

vacevedo@alemana.cl

Dra. Lidia Valenzuela

Lidia.valenzuela@redsalud.gov.cl

Dra. Patricia Verdugo

Pverdugo33@hotmail.com

Dra. Mónica Varas

monicavaraspalma@gmail.com

\section{Referencias bibliográficas}

1.- Dhama K, Khan S, Tiwari R, Sircar S, Bhat S, Malik Y S, et al. 2020. Coronavirus disease 2019-COVID-19. Clin Microbiol Rev 2020; 33:e0028-20. doi: 10.1128/CMR.00028-20.

2.- Zhu N, Zhang D, Wang W, Li X, Yang B, Song $\mathrm{J}$, et al. A novel coronavirus from patients with pneumonia in China, 2019. N Engl J Med 2020; 382: 727-33. doi: 10.1056/NEJMoa2001017.

3.- World Health Organization. (2009). Pandemic influenza preparedness and response: A WHO Guidance Document. Geneva. https://www. who.int/influenza/resources/documents/ pandemic_guidance_04_2009/en/

4.- She J, Liu L, Liu W. COVID-19 epidemic: disease characteristics in children. J Med Virol 2020; 92 (7): 747-54. doi:10.1002/jmv.25807.

5.- Pavone P, Ceccarelli M, Taibi R, La Rocca G, Nunnari G. Outbreak of COVID-19 infection in children: fear and serenity. Eur Rev Med Pharmacol Sci 2020; 24 (8): 4572-5. doi: 10.26355/eurrev_202004_21043.

6.- Zimmermann P, Curtis N. Coronavirus infections in children including COVID-19: An overview of the epidemiology, clinical features, diagnosis, treatment and prevention options in children. Pediatr Infect Dis J 2020; 39 (5): 355 68. doi:10.1097/INF.0000000000002660.

7.- Han YN, Feng ZW, Sun LN, Ren XX, Wang
H, Xue YM, et al. A comparative-descriptive analysis of clinical characteristics in 2019coronavirus-infected children and adults. J Med Virol 2020; 1-7. doi: 10.1002/jmv.25835.

8.- Shen K, Yang Y, Wang T, Zhao D , Jiang Y, Jin $\mathrm{R}$, et al. Diagnosis, treatment, and prevention of 2019 novel coronavirus infection in children: experts' consensus statement. World J Pediatr. 2020; 16 (3): 223-31. doi: 10.1007/s12519-02000343-7.

9.- Licciardi F, Pruccoli G, Denina M, Parodi E, Taglietto M, Rosati S, et al. SARS-CoV-2Induced Kawasaki-like hyperinflammatory syndrome: a novel COVID phenotype in children. Pediatrics May 2020, e20201711; doi: https://doi.org/10.1542/peds.2020-1711.

10.- Chiotos K, Bassiri H, Behrens E M, Blatz A M, Chang J, Diorio C, et al. Multisystem inflammatory syndrome in children during the coronavirus 2019 pandemic: a case series. J Pediatric Infect Dis Soc 2020; 9(3): 393-8. doi:10.1093/jpids/piaa069.

11.- Feldstein LR, Rose EB, Horwitz SM, Collins JP, Newhams MM, Son MBF, et al. Multisystem Inflammatory Syndrome in U.S. children and adolescents. N Engl J Med 2020; 383: 334-46. doi: 10.1056/NEJMoa2021680.

12.- Torres J P, Izquierdo G, Acuña M, Pavez D, Reyes F, Fritis A, et al. Multisystem inflammatory syndrome in children (MIS-C):
Report of the clinical and epidemiological characteristics of cases in Santiago de Chile during the SARS-CoV-2 pandemic. Intern J Infect Dis 100 (2020): 75-81. https://doi. org/10.1016/j.ijid.2020.08.062.

13.- CDC (2020). Information for healthcare providers about Multisystem Inflammatory Syndrome in Children (MIS-C). https://www. cdc.gov/mis-c/hcp/

14.- Recomendaciones sobre el manejo clínico de la infección por "nuevo coronavirus" SARS-CoV-2. Grupo de trabajo de Asociación Española de Pediatría (AEP). Cristina Calvo \& cols. An Pediatr (Barc).

15.- Recomendaciones de Manejo Farmacológico en Pacientes Pediátricos con Infección por SARSCoV-2 (COVID-19) de la Sociedad Chilena de Infectología, Rama de Cuidados Intensivos Pediátricos de la SOCHIPE y Sociedad de Neumología Pediátrica. 03 abril 2020. https:// sochinep.com/site/docs/recomendaciones sochineps.pdf.

16.- Manejo de Pacientes Pediátricos Cursando Neumonia Grave/SDRA por SARS-CoV-2 de la Sociedad Chilena de Pediatría y la Sociedad de Cuidados Intensivos Pediátricos. https://www. sochipe.cl/subidos/revista1/docs/1587661966EE\%20N108\%20-\%20FINAL.pdf.

17.- Recomendaciones frente a la infección por SARS-CoV-2 (COVID-19) en pacientes 
pediátricos con enfermedades oncológicas y receptores de transplante de progenitores hematopoyéticos. Programa PINDA, Chile. 03 Abril 2020. https://slipe.org/web/wp-content/ uploads/2020/04/COVID-en-IC-para-SLIPE-7de-abril-2020.pdf.

18.- Whittaker E, Bamford A, Kenny J, Kaforou M, Jones CE, Shah P, et al. Clinical characteristics of 58 children with a pediatric inflammatory multisystem syndrome temporally associated with SARS-CoV-2. JAMA. 2020; 324(3): 25969. doi:10.1001/jama.2020.10369.

19.- Ramcharan T, Nolan O, Lai CY, Prabhu N, Krishnamurthy R, Richter AG, et al. Paediatric inflammatory multisystem. syndrome: temporally associated with SARS-CoV-2 (PIMS-TS): cardiac features, management and short-term outcomes at a UK tertiary paediatric hospital. Pediatr Cardiol 2020; 1-11. doi: 10.1007/s00246-020-02391-2.

20.- Ulloa-Gutiérrez R, Ivankovich-Escoto G, Yamazaki-Nakashimada M A. Multisystem inflammatory syndrome associated with COVID19 in children and adolescents: calling for diagnosis. Rev Chilena Infectol 2020; 37 (3): 202-4. doi: http://dx.doi.org/10.4067/ s0716-10182020000300199. 